\title{
Bounds on the Coupling Constant in Two Dimensional Boson Models
}

\author{
A. L. Carey ${ }^{1}$, M. A. Lohe ${ }^{2}$, and D. M. O'Brien ${ }^{2}$ \\ ${ }^{1}$ Department of Pure Mathematics, University of Adelaide, Adelaide, South Australia 5000 \\ 2 Department of Mathematical Physics, University of Adelaide, Adelaide, South Australia, 5000
}

\begin{abstract}
We consider two dimensional boson field theories with an interaction potential $\mathscr{V}(\phi)$. We show how to define a cut-off, renormalised Hamiltonian for a certain class of non-polynomial $\mathscr{V}(\phi)$, which are defined via an integral transform. We formulate precisely a variational argument devised by Coleman, obtaining a constraint on the coupling constant of the theory with general $\mathscr{V}(\phi)$, and illustrate the argument with several examples.
\end{abstract}

\section{Introduction}

In this note we will consider boson field theories obtained by quantising the classical system with Lagrangian density

$$
\mathscr{L}=-\frac{1}{2}\left(\partial_{\mu} \phi\right)\left(\partial^{\mu} \phi\right)+\lambda^{-2} \mathscr{V}(\lambda \phi)
$$

for a range of potentials $\mathscr{V}$. The Hamiltonian density corresponding to $\mathscr{L}$ is

$$
\mathscr{H}=\mathscr{H}^{0}+\lambda^{-2} \mathscr{V}(\lambda \phi)
$$

with

$$
\mathscr{H}^{0}=\frac{1}{2}\left[\pi^{2}+(\nabla \phi)^{2}\right],
$$

where $\pi$ is the field conjugate to $\phi$. We will allow only two space-time dimensions. Our aims are two-fold, namely:

(1) to define a cut-off, renormalised Hamiltonian for a range of non-polynomial interactions $\mathscr{V}$;

(2) to reformulate within the framework of constructive field theory a variational argument due to Coleman [1], which places constraints on the various parameters appearing in $\mathscr{V}$.

Let us begin by reviewing the argument which Coleman applied to the sineGordon model, defined by the potential

$$
\mathscr{V}(\phi)=k(1-\cos \phi), \quad k>0 .
$$


(1) Coleman adopted an unspecified representation of the canonical commutation relations (CCR), defined

$$
\phi_{A}^{ \pm}=\frac{1}{2}\left(\phi \mp i A^{-1} \pi\right), \quad \pi_{A}^{ \pm}=-\frac{1}{2}(\pi \pm i A \phi),
$$

where

$$
A^{2}=-\nabla^{2}+\alpha^{2}
$$

and regarded $\phi_{A}^{+}$and $\pi_{A}^{+}$as creation operators for a free field of mass $\alpha$, and $\phi_{A}^{-}$ and $\pi_{A}^{-}$as the corresponding annihilation operators. To any function of $\phi$ and $\pi$, such as $\mathscr{H}$, there corresponds a normally ordered form, denoted by $: \mathscr{H}:{ }_{A}$, obtained by inserting

$$
\begin{aligned}
\phi & =\phi_{A}^{+}+\phi_{A}^{-}, \\
-\pi & =\pi_{A}^{+}+\pi_{A}^{-}
\end{aligned}
$$

into the expression for $\mathscr{H}$ and arranging the annihilation operators to the right of the creation operators. The normally ordered forms obtained with different choices for the mass parameter are related by the reordering formulae,

in which

$$
\begin{aligned}
: \mathscr{H}^{0}:_{A} & =: \mathscr{H}^{0}:_{B}+\left(\beta^{2}-\alpha^{2}\right) / 8 \pi \\
: \mathscr{V}:_{A} & =: \exp \left[\frac{1}{8 \pi} \log \left(\frac{\alpha^{2}}{\beta^{2}}\right) \frac{d^{2}}{d \phi^{2}}\right] \mathscr{V}:_{B},
\end{aligned}
$$

$$
A^{2}=-\nabla^{2}+\alpha^{2}, \quad B^{2}=-\nabla^{2}+\beta^{2},
$$

and the constants are correct to leading order in $\Lambda$, the momentum cut-off.

(2) Because there is only one space dimension, the Hamiltonian density can be renormalised simply by writing it in normally ordered form, so Coleman chose $: \mathscr{H}:_{A}$, normally ordered with respect to a fixed mass $\alpha$, as the Hamiltonian density of the sine-Gordon model.

(3) According to the Rayleigh-Ritz variational principle, the infimum of the spectrum of $: \mathscr{H}:{ }_{A}$ is bounded above by

$$
\left(v,: \mathscr{H}:{ }_{A} v\right) /(v, v)
$$

for all $v$ in the domain of $: \mathscr{H}:{ }_{A}$. Coleman chose for the trial vector the vacuum vector for particles of mass $\beta$, defined as the vector $v_{B}$ annihilated by $\phi_{B}^{-}$and $\pi_{B}^{-}$,

$$
\begin{gathered}
\phi_{B}^{-} v_{B}=0, \quad \pi_{B}^{-} v_{B}=0, \\
B^{2}=-\nabla^{2}+\beta^{2},
\end{gathered}
$$

and used the reordering formulae to compute

$$
\left(v_{B},: \mathscr{H}:_{A} v_{B}\right) /\left(v_{B}, v_{B}\right)=\left(\beta^{2}-\alpha^{2}\right) / 8 \pi-k \lambda^{-2}\left(\beta^{2} / \alpha^{2}\right)^{\lambda^{2} / 8 \pi}
$$

Coleman observed that the right hand side of (1.1) is only bounded below as a function of $\beta$ if $\lambda^{2} \leqq 8 \pi$, and concluded that this constraint is necessary if the energy is to be bounded below and the theory is to have physical content.

Coleman's argument is particularly useful, because it is non-perturbative and 
provides a priori bounds on the coupling constant, so we attempted to extend it to a wider class of potentials. Now the key to the argument is the ability to evaluate

$$
\left(v_{B},: \mathscr{H}:{ }_{A} v_{B}\right) /\left(v_{B}, v_{B}\right)=\left(\beta^{2}-\alpha^{2}\right) / 8 \pi
$$

and

$$
\left(v_{B},: \mathscr{V}:_{A} v_{B}\right) /\left(v_{B}, v_{B}\right)=\left.\exp \left[\frac{1}{8 \pi} \log \left(\frac{\alpha^{2}}{\beta^{2}}\right) \frac{d^{2}}{d \phi^{2}}\right] \mathscr{V}(\phi)\right|_{\phi=0} .
$$

This is a simple step for the sine-Gordon model because $\mathscr{V}$ is an exponential function, so the obvious extensions are to potentials which have a finite Fourier series,

$$
\mathscr{V}(\phi)=\frac{1}{2 \pi} \sum_{k=1}^{n} a_{k} e^{-i s_{k} \phi},
$$

and to potentials which have a Fourier integral representation,

$$
\mathscr{V}(\phi)=\frac{1}{2 \pi} \int_{\mathbb{R}} e^{-i s \phi_{\mathscr{r}} \hat{V}}(s) d s .
$$

More generally, we can allow potentials which have an integral representation with an exponential kernel,

$$
\mathscr{V}(\phi)=\frac{1}{2 \pi i} \int_{\Gamma} e^{-i s \phi} \hat{\mathscr{V}}(s) d s,
$$

where $\Gamma$ denotes a closed contour in the complex plane. Indeed, both (1.2) and (1.3) are special cases of (1.4). For such potentials,

$$
\begin{aligned}
\lambda^{-2}: \mathscr{V}(\lambda \phi):_{A} & =\frac{\lambda^{-2}}{2 \pi i} \int_{\Gamma}: e^{-i s \lambda \phi}:_{A} \hat{\mathscr{V}}(s) d s \\
& =\frac{\lambda^{-2}}{2 \pi i} \int_{\Gamma}\left(\frac{\beta^{2}}{\alpha^{2}}\right)^{s^{2} \lambda^{2} / 8 \pi}: e^{-i s \lambda \phi}:_{B} \hat{\mathscr{V}}(s) d s,
\end{aligned}
$$

so we find that

$$
\frac{\left(v_{B},: \mathscr{H}:{ }_{A} v_{B}\right)}{\left(v_{B}, v_{B}\right)}=\frac{\beta^{2}-\alpha^{2}}{8 \pi}+\frac{\lambda^{-2}}{2 \pi i} \int\left(\frac{\beta^{2}}{\alpha^{2}}\right)^{s^{2} \lambda^{2} / 8 \pi} \hat{\mathscr{V}}(s) d s .
$$

If the right hand side of (1.5) is not bounded below as a function of $\beta$ for some value of $\lambda$, then we can conclude that the value of $\lambda$ is inadmissible.

Unfortunately there is a gap in Coleman's argument because the mass $\beta$ vacuum state is not contained in the representation space of the CCR chosen by Coleman. What then is the meaning of $\left(v_{B},: \mathscr{H}_{A} v_{B}\right) /\left(v_{B}, v_{B}\right)$, and how does the familiar Rayleigh-Ritz variational argument apply? Again, is the bound obtained by Coleman dependent upon the representation he chose for the CCR? Our aim is to answer these questions and to show that a careful modification of Coleman's argument does provide $a$ priori bounds on the coupling constant for a range of potentials. 
In our reformulation of Coleman's argument, three operators will keep appearing,

$$
\begin{aligned}
& A^{2}=-\nabla^{2}+\alpha^{2}, \\
& B^{2}=-\nabla^{2}+\beta^{2}, \\
& C^{2}=-\nabla^{2}+\gamma^{2} .
\end{aligned}
$$

(1) We adopt the $C$-representation of the CCR, corresponding to a free field $\phi$ and its conjugate $\pi$ with mass $\gamma$. We impose a momentum cut-off $\Lambda$ on $\phi$ and $\pi$, thereby producing new fields $\phi_{\Lambda}$ and $\pi_{\Lambda}$, and construct

and

$$
\begin{aligned}
\mathscr{H}_{\Lambda}^{0}(x) & =\mathscr{H}^{0}\left(\phi_{\Lambda}(x), \pi_{\Lambda}(x)\right), \\
\mathscr{V}_{\Lambda}(x) & =\lambda^{-2} \mathscr{V}\left(\lambda \phi_{\Lambda}(x)\right),
\end{aligned}
$$

$$
\mathscr{H}_{\Lambda}(x)=\mathscr{H}_{\Lambda}^{0}(x)+\mathscr{V}_{\Lambda}(x) .
$$

From the Hamiltonian density we construct the Hamiltonian with a box cut-off $L$,

$$
\begin{aligned}
& H_{\Lambda L}^{0}=\int_{-L}^{L} \mathscr{H}_{\Lambda}^{0}(x) d x, \\
& V_{\Lambda L}=\int_{-L}^{L} \mathscr{V}_{\Lambda}(x) d x
\end{aligned}
$$

and

$$
H_{\Lambda L}=H_{\Lambda L}^{0}+V_{\Lambda L} .
$$

We emphasise that we have included both cut-offs, $\Lambda$ and $L$, in the free Hamiltonian as well as the interaction term, contrary to the usual practice in constructive field theory of applying the cut-offs only to the interaction.

(2) Next we construct the mass $\alpha$ annihilation and creation operators,

$$
\begin{array}{rrr}
\phi_{\Lambda A}^{ \pm}=\frac{1}{2}\left[\phi_{\Lambda} \mp i A^{-1} \pi_{A}\right], & \phi_{A}=\phi_{\Lambda A}^{+}+\phi_{\Lambda A}^{-}, \\
\pi_{A A}^{ \pm}=-\frac{1}{2}\left[\pi_{\Lambda} \pm i A \phi_{\Lambda}\right], & -\pi_{A}=\pi_{A A}^{+}+\pi_{\Lambda A}^{-},
\end{array}
$$

and normally order both $\mathscr{H}_{A}$ and $H_{A L}$ by arranging the $A$-annihilation operators to the right of the $A$-creation operators, to produce: $\mathscr{H}_{A}:_{A}$ and $: H_{A L}:_{A}$.

(3) We assume that : $H_{A L}:{ }_{A}$ is essentially self adjoint on the domain $G$ defined in Sect. 2. (This assumption is necessary for step (4).) For results relevant to this see [8].

(4) Lastly, we prove that there is a sequence of vectors $v_{B}^{(n)}$ in the domain of $: \overline{H_{A L}{ }_{A}}$ (the closure of $: H_{A L}:_{A}$ ) which approximates the mass $\beta$ vacuum and satisfies

$$
\inf _{n} \frac{\left(v_{B}^{(n)},: H_{\Lambda L}:_{A} v_{B}^{(n)}\right)_{C}}{\left(v_{B}^{(n)}, v_{B}^{(n)}\right)_{C}} \leqq 2 L\left[\frac{\beta^{2}-\alpha^{2}}{8 \pi}+\frac{\lambda^{-2}}{2 \pi i} \int_{\Gamma}\left(\frac{\beta^{2}}{\alpha^{2}}\right)^{s^{2} \lambda^{2} / 8 \pi} \hat{V}(s) d s+0\left(\Lambda^{-1}\right)\right]
$$

If we can now prove that the right hand side of (1.6) is unbounded below for certain choices of $\lambda$, then we can conclude that $: H_{A L}:_{A}$ is unbounded below for any fixed 
box cut-off $L$ and all sufficiently large momentum cut-offs $\Lambda$. This reproduces Coleman's argument.

Whether this proves that the Hamiltonian (without cut-offs) cannot be bounded below is contentious. Firstly, the existence of a Wightman field theory with dynamics corresponding to the classical Hamiltonian is unproven (except for the polynomial and sine-Gordon interactions). Secondly, even if the Hamiltonian exists, as the generator of time translations in the Wightman field theory, it is not clear that it can be obtained by removing the cut-offs from $: H_{A L}:_{A}$. Indeed, if we naively lift the momentum cut-off, so that $\phi_{A}$ and $\pi_{A}$ become local, then the results of Epstein, Glaser and Jaffe [2] might be applicable, which show that $\mathscr{H}^{0}$ cannot be positive definite and suggest (but do not prove) that both $\mathscr{H}^{0}$ and $H_{L}^{0}$ might be unbounded below and that $H_{L}^{0}$ might have continuous spectrum. However, Coleman's argument only requires that the Hamiltonian should be unbounded below whenever $: H_{A L}:_{A}$ is unbounded below. This point is possibly not difficult to check in a particular model, given the existence of the Hamiltonian, but we do not intend to pursue it here.

At first sight our argument may appear unnecessarily complicated, for we have used three operators $A, B, C$, when the usual procedure is to set $A=C$. However, this specialisation does not simplify the calculations, so we will carry through the argument for general $A$.

Finally, we remark that there may be other ways of recovering Coleman's conclusions, using different cut-offs or an alternative approach, but the setting which seemed clearest to us was the configuration space representation of the canonical commutation relations, described by Glimm and Jaffe [3]. In the first section of the paper we collect some elementary properties of this representation. This done, we introduce the class of potentials to which our argument will apply. Then comes the variational argument, which we conclude with some examples.

We note that Chodos and Klein [4] have also applied Coleman's argument to the potential $e^{\phi^{*} \phi}$. For general discussion of non-polynomial interaction see Hфegh-Krohn [8] and for rigorous results on sine-Gordon see Fröhlich [9].

\section{Notation and Preliminaries}

We let $S$ denote the space of real-valued test functions on $\mathbb{R}$ of fast decrease and write $u, v \rightarrow\langle u, v\rangle$ for the Euclidean inner product of elements $u, v \in S$. The dual of $S$ we denote by $Q$, and the pairing between $Q$ and $S$ by $\langle q, u\rangle, q \in Q, u \in S$.

To each $u \in S$, there corresponds a coordinate function on $Q, u: q \rightarrow\langle q, u\rangle \in \mathbb{R}$, which we also denote by $u$. Given coordinates $u_{1}, \ldots, u_{n}$ on $Q$ and any complex valued function $f$ in $n$ variables, then we form the cylinder function $f: q \rightarrow f\left(u_{1}(q)\right.$, $\left.u_{2}(q), \ldots, u_{n}(q)\right)$ on $Q$ with base $U=\operatorname{span}\left\{u_{1}, u_{2}, \ldots, u_{n}\right\}$.

If $f$ is a differentiable cylinder function then we write for its derivative with respect to a coordinate $v$,

$$
\left(\partial_{v} f\right)(q)=\sum_{k=1}^{n}\left\langle v, u_{k}\right\rangle\left(\partial_{k} f\right)\left(u_{1}(q), u_{2}(q), \ldots, u_{n}(q)\right) .
$$

The set of cylinder functions is an algebra over $\mathbb{C}$ in which addition and multiplica- 
tion are defined pointwise. We let $P$ denote the subalgebra consisting of the polynomial functions on $Q$, and $G$ denote the cylinder functions with exponential growth of order 2 and minimal type. This latter is defined to be the set of $C^{\infty}$ cylinder functions $f$ which satisfy

$$
\left|\partial_{v_{1}} \ldots \partial_{v_{m}} f(q)\right| \leqq C \exp \varepsilon \sum_{i=1}^{n}\left\langle q, u_{i}\right\rangle^{2}
$$

for any $\varepsilon>0$ and coordinates $v_{1}, v_{2}, \ldots, v_{m}$. The constant $C$ may depend upon $\varepsilon$ and the coordinates. It is straightforward to verify that $G$ is also an algebra.

Given any real, symmetric, positive-definite operator $C$ on $S$ we define

$$
\langle u, v\rangle_{C}=\left\langle u, C^{-1} v\right\rangle \text { and }\|u\|_{C}=\langle u, u\rangle_{C}^{1 / 2} .
$$

We let $v_{C}$ denote the corresponding Gaussian measure on $Q[5]$ whose Fourier transform is

$$
\int_{Q} \exp (2 i s\langle q, u\rangle) d v_{c}(q)=\exp \left(-s^{2}\|u\|_{c}^{2}\right) .
$$

Finally, we note that $P$ and $G$ are densely embedded subalgebras of $L^{2}\left(Q, v_{C}\right)$ for all $C$. More details on the above may be found in [5].

We will later need certain integration formulae which are summarised in the following lemma.

Lemma 1. Suppose that: dent;

(1) $u_{1}, u_{2}, \ldots, u_{n}$ are arbitrary elements of $S$, not necessarily linearly indepen-

(2) $N_{r s}=\left\langle u_{r}, u_{s}\right\rangle_{C}$

(3) $M_{r s}$ is a real, symmetric matrix, not necessarily positive definite; Let

(4) $z_{1}, \ldots, z_{n}$ are parameters, not necessarily real.

$$
I=\int_{Q} \exp \left[\sum_{r, s=1}^{n} u_{r}(q) M_{r s} u_{s}(q)+2 \sum_{r=1}^{n} z_{r} u_{r}(q)\right] d v_{C}(q)
$$

and

$$
J=\int_{Q} p\left(u_{1}(q), \ldots, u_{n}(q)\right) \exp \left[\sum_{r, s=1}^{n} u_{r}(q) M_{r s} u_{s}(q)+2 \sum_{r=1}^{n} z_{r} u_{r}(q)\right] d v_{c}(q),
$$

where $p$ is a polynomial in $n$ variables. Then the integrals are convergent if and only if $\mathrm{N}^{+}-\mathrm{M}$ is positive definite on the orthogonal complement of the kernel of $N$, where $\mathrm{N}^{+}$denotes the Moore-Penrose generalised inverse of $N$. When this condition is satisfied,

$$
I=\operatorname{det}^{-1 / 2}(1-M N) \exp \left[\sum_{r, s=1}^{n} z_{r}\left[N(1-M N)^{-1}\right]_{r s} z_{s}\right]
$$

and

$$
J=p\left(\frac{1 \partial}{2 \partial z_{1}}, \ldots, \frac{1 \partial^{n}}{2 \partial z_{n}}\right) I
$$


The proof is straightforward, so we omit it.

The condition for convergence of the integrals can be difficult to check when the matrices are large, but one situation (which we will later encounter) is covered by the following lemma.

Lemma 2. Suppose that $u_{1}, u_{2}, \ldots, u_{n}$ are linearly independent, so that $N>0$. if

$$
M_{r s}=0 \quad \text { whenever } r>k \text { or } s>k
$$

then

$$
N^{-1}-M>0 \quad \text { if and only if } N_{k}^{-1}-M_{k}>0,
$$

where $M_{k}$ and $N_{k}$ denote $M$ and $N$ truncated to $k$ rows and columns.

Proof. The 'only if' part of the lemma is obvious, and the 'if' part is established by induction on $n$. By hypothesis, the result is true when $n=k$. Suppose then that $N_{\ell}^{-1}-M_{\ell}>0$ for some $\ell \geqq k$. The augmented matrix $N_{\ell+1}$ is positive because $u_{1}, u_{2}, \ldots, u_{\ell+1}$ are linearly independent, so if we write

$$
N_{\ell+1}=\left[\begin{array}{cc}
N_{\ell} & \mathbf{a}_{\ell} \\
\mathbf{a}_{\ell}^{*} & p_{\ell}
\end{array}\right], \quad M_{\ell+1}=\left[\begin{array}{cc}
M_{\ell} & 0 \\
0 & 0
\end{array}\right],
$$

where

$$
\left(\mathbf{a}_{\ell}\right)_{j}=\left\langle u_{j}, u_{\ell+1}\right\rangle_{c}, \quad j=1,2, \ldots, \ell
$$

and

$$
p_{\ell}=\left\langle u_{\ell+1}, u_{\ell+1}\right\rangle_{c}
$$

then it follows from Lemma 3 below that $N_{\ell+1}^{-1}-M_{\ell+1}>0$.

Lemma 3. Let $P_{\ell}$ and $Q_{\ell}$ be hermitian matrices with $P_{\ell}>0$ and $R_{\ell}=P_{\ell}^{-1}-Q_{\ell}>0$. If $P_{\ell}$ and $Q_{\ell}$ are augmented by the addition of a row and a column,

$$
P_{\ell+1}=\left[\begin{array}{cc}
P_{\ell} & \mathbf{a}_{\ell} \\
\mathbf{a}_{\ell}^{*} & p_{\ell}
\end{array}\right], \quad Q_{\ell+1}=\left[\begin{array}{cc}
Q_{\ell} & 0 \\
0 & 0
\end{array}\right]
$$

such that $P_{\ell+1}>0$, then $R_{\ell+1}=P_{\ell+1}^{-1}-Q_{\ell+1}>0$.

This is a simple verification so we omit the proof.

\section{Integral Representation of Cylinder Functions in $\mathbf{G}$}

Let $f \in G$ have base $U$. The $C$-Fourier transform of $f$ is the map $\tilde{f}: U \rightarrow U$ where

$$
\tilde{f}(u)=\int_{Q} \exp \left(2 i\langle q, u\rangle+\langle u, u\rangle_{C}\right) f(q) d v_{C}(q) .
$$

We parametrise $U$ by assuming, without loss of generality, that $u_{1}, u_{2}, \ldots, u_{n}$ are $C$-orthonormal, so that we can represent a general element of $U$ in the form $u=\mathbf{z} \cdot \mathbf{u}$, where $\mathbf{u}=\left(u_{1}, u_{2}, \ldots, u_{n}\right), \mathbf{z}=\left(z_{1}, z_{2}, \ldots, z_{n}\right)$, and $z_{i}=\left\langle u, u_{i}\right\rangle_{C}$. Then $\tilde{f}$ can be regarded as a function of $\mathbf{z}$ and

$$
\exp \left(-|\mathbf{z}|^{2}\right) \tilde{f}(\mathbf{z})=\pi^{-n / 2} \int_{\mathbb{R}^{n}} f(\mathbf{t}) \exp \left[2 i \mathbf{z} \cdot \mathbf{t}-|\mathbf{t}|^{2}\right] d \mathbf{t} .
$$


The function $g(\mathbf{t})=f(\mathbf{t}) \exp \left(-|\mathbf{t}|^{2}\right)$ belongs to the space $S_{1 / 2}$ of Gelfand and Shilov [2] because $\left|g^{(\mathbf{k})}(\mathbf{t})\right| \leqq C_{\mathbf{k}} \exp \left(-a \mid \mathbf{t}^{2}\right)$ with $0<a<1$. Moreover the Fourier transform of $S_{1 / 2}$ is $S^{1 / 2}$, consisting of entire functions of order 2, so $\tilde{f}$ must also be entire with order $r \leqq 2$.

Every such function has a Borel transform [7]

$$
f^{\#}(\mathbf{s})=r \int_{0}^{\infty}\left[\prod_{i=1}^{n} z_{i}^{r-1} \exp \left(-z_{i}^{r}\right) / s_{i}\right] \tilde{f}\left(z_{1} / s_{1}, z_{2} / s_{2}, \ldots, z_{n} / s_{n}\right) d \mathbf{z},
$$

which is holomorphic for all sufficiently large s. Furthermore, if $\Gamma$ denotes a simple, closed contour which encloses the singularities of $f^{H}$, then

$$
\tilde{f}(\mathbf{z})=\frac{1}{(2 \pi i)^{n}} \int_{\Gamma}\left[\prod_{i=1}^{n} E_{r}\left(z_{i} s_{i}\right)\right] f^{\#}(\mathbf{s}) d \mathbf{s},
$$

where $E_{r}$ is the Mittag-Leffler function,

Thus, we find that

$$
E_{r}(z)=\sum_{k=0}^{\infty} \frac{z^{k}}{\Gamma(1+k / r)} .
$$

$$
f(\mathbf{t})=\frac{1}{(2 \pi i)^{n}} \int_{\Gamma} k_{r}(\mathbf{t}, \mathbf{s}) f^{H}(\mathbf{s}) d \mathbf{s},
$$

in which

$$
k_{r}(\mathbf{t}, \mathbf{s})=\sum_{\mathbf{m}=0}^{\infty} H_{\mathbf{m}}(\mathbf{t}) \prod_{i=1}^{n} \frac{\left(-i s_{i} / 2\right)^{m_{i}}}{\Gamma\left(1+m_{i} / r\right)}, \quad \mathbf{m}=\left(m_{1}, m_{2}, \ldots, m_{n}\right),
$$

and $H_{\mathbf{m}}$ denotes the $\mathbf{m}^{\text {th }}$ order Hermite polynomial,

$$
H_{\mathbf{m}}(\mathbf{t})=e^{|\mathbf{t}|^{2}} \prod_{i=1}^{n}\left(-\frac{\partial}{\partial t_{i}}\right)^{m_{i}} e^{-|t|^{2}}
$$

In particular,

so

$$
k_{1}(\mathbf{t}, \mathbf{s})=\exp \left(\frac{1}{4}|\mathbf{s}|^{2}-i \mathbf{t} \cdot \mathbf{s}\right),
$$

$$
f(\mathbf{t})=\frac{1}{(2 \pi i)^{n}} \int_{\Gamma} e^{-i \mathbf{t} \cdot \mathbf{s}} \hat{f}(\mathbf{s}) d \mathbf{s},
$$

where

$$
\hat{f}(\mathbf{s})=e^{1 / 4|\mathbf{s}|^{2}} f^{H}(\mathbf{s}) .
$$

We have shown that $f$ has the integral representation

$$
f(q)=\frac{1}{(2 \pi i)^{n}} \int_{\Gamma} k_{r}(\langle q, \mathbf{u}\rangle, \mathbf{s}) f^{H}(\mathbf{s}) d \mathbf{s}
$$

in which $\Gamma$ is a simple, closed, bounded contour in $\mathbb{C}^{n}$ and the parameter $r$ is the order of growth of the Fourier transform $\tilde{f}$ of $f$. In particular, when $f$ is a function 
of exponential type, so that $r=1$, then (3.1) reduces to

$$
f(q)=\frac{1}{(2 \pi i)^{n}} \int_{\Gamma} \exp (-i \mathbf{s} \cdot\langle q, \mathbf{u}\rangle) \hat{f}(\mathbf{s}) d \mathbf{s}
$$

\section{Normal Ordering}

We introduce the $C$-representation of the canonical commutation relations on $L^{2}\left(Q, v_{c}\right)[5]$. For each $u \in S$, we let $\phi_{u}$ and $\pi_{u}$ denote the operators

$$
\phi_{u}: f \rightarrow u f, \pi_{u}: f \rightarrow-i \partial_{u} f+i(C u) f, \quad f \in G .
$$

These operators leave $G$ invariant, are symmetric, and satisfy $\left[\phi_{u}, \pi_{v}\right]=i\langle u, v\rangle$ on $G$.

If $K$ is an operator on $S$, then we write $K \phi_{u}=\phi_{K u}$ and $K \pi_{u}=\pi_{K u}$. So in particular with $A^{2}=-\nabla^{2}+\alpha^{2}$ we may define

$$
\phi_{u}^{ \pm}=\frac{1}{2}\left[\phi_{u} \mp i A^{-1} \pi_{u}\right], \quad \pi_{u}^{ \pm}=-\frac{1}{2}\left[\pi_{u} \pm i A \phi_{u}\right] .
$$

Given any polynomial $p\left(\phi_{u}, \pi_{v}\right)$ in $\phi_{u}$ and $\pi_{v}$, then we can write $p=p\left[\phi_{u}^{+}+\phi_{u}^{-}\right.$, $-\pi_{v}^{+}-\pi_{v}^{-}$) and arrange the annihilation operators to the right of the creation operators in the expansion of $p$. We call this the $A$-normally ordered form of $p$ and denote it by $: p:_{A}$.

In order to extend this procedure to non-polynomial functions of $\phi_{u}$, we define the normally ordered form for the exponential function on $Q$ and then extend the definition to those functions in $G$ with Fourier transforms of exponential type. Given coordinate functions $u_{1}, u_{2}, \ldots, u_{n}$ on $Q$ and possibly complex $s_{1}, s_{2}, \ldots, s_{n}$, we define

$$
: \exp (-i \mathbf{s} \cdot \mathbf{u}):_{A}=\exp (-i \mathbf{s} \cdot \mathbf{u}) \exp \left(\frac{1}{4} \sum_{i, j=1}^{n} s_{i}\left\langle u_{i}, u_{j}\right\rangle s_{j}\right),
$$

where $\mathbf{s}=\left(s_{1}, s_{2}, \ldots, s_{n}\right)$ and $\mathbf{u}=\left(u_{1}, u_{2}, \ldots, u_{n}\right)$. Note that

$$
\int_{Q}: \exp (-i \mathbf{s} \cdot \mathbf{u}):_{A}(q) d v_{A}(q)=1
$$

If $f \in G$ has the representation (3.2) we may define

$$
: f:_{A}(q)=\frac{1}{(2 \pi i)^{n}}{ }_{\Gamma}: \exp (-i \mathbf{s} \cdot \mathbf{u}):_{A}(q) \hat{f}(\mathbf{s}) d \mathbf{s} .
$$

For polynomial functions this gives us two definitions of normal ordering but we will see later that they coincide. Finally, if $f$ is a cylinder function in $G$, then $: f:_{A}$ also lies in $G$.

A similar definition of normal ordering for a more restrictive class of potentials is given by $\mathrm{H} \phi$ egh-Krohn [8].

\section{Formal Calculations}

We now have the machinery to analyse the steps of the variational argument, but before doing so we will sketch the underlying formal calculations. 
If we were to let the smearing function shrink to a $\delta$ function, then the fields $\phi(x)$ and $\pi(x)$ on $Q$ space would become the operators

$$
\begin{aligned}
& \phi(x) f(q)=q(x) f(q), \\
& \pi(x) f(q)=\left[-i \frac{\partial}{\partial q(x)}+i C q(x)\right] f(q),
\end{aligned}
$$

with corresponding annihilation and creation operators,

$$
\begin{aligned}
\phi_{B}^{-}(x) f(q) & =\frac{1}{2}\left[q(x)-B^{-1} C q(x)+B^{-1} \frac{\partial}{\partial q(x)}\right] f(q), \\
\pi_{B}^{-}(x) f(q) & =\frac{i}{2}\left[\frac{\partial}{\partial q(x)}-C q(x)+B q(x)\right] f(q) .
\end{aligned}
$$

They act on the space of functions of $q$, equipped with the inner product,

$$
(f, g)_{C}=\int_{Q} \overline{f(q)} g(q) e^{-(q, C q)} d q,
$$

where

$$
(q, C q)=\int q(x)\left[\int C(x-y) q(y) d y\right] d x,
$$

and

$$
C(x-y)=\frac{1}{2 \pi} \int e^{i k(y-x)} \sqrt{k^{2}+\gamma^{2}} d k
$$

is the kernel of $C$. The annihilation operators commute,

$$
\left[\phi_{B}^{-}(x), \pi_{B}^{-}(y)\right]=0,
$$

so it ought to be possible to find a simultaneous solution of the equations,

$$
\begin{aligned}
& \phi_{B}^{-}(x) f(x)=0, \\
& \pi_{B}^{-}(x) f(x)=0 .
\end{aligned}
$$

Indeed, the formal solution is

$$
f(x)=\exp \frac{1}{2} q(x)[(C-B) q](x) .
$$

Furthermore,

$$
\begin{aligned}
v_{B} & =\prod f(x) \\
& =\exp \frac{1}{2} \int d x q(x)[(C-B) q](x) \\
& =\exp \frac{1}{2}(q,(C-B) q)
\end{aligned}
$$

will satisfy

$$
\phi_{B}^{-}(x) v_{B}=0, \quad \pi_{B}^{-}(x) v_{B}=0
$$

for all $x$. The vector $v_{B}$ is the $B$-vacuum vector.

Our aim is to compute

$$
\left(v_{B},: H_{A L}:_{A} v_{B}\right)_{C} /\left(v_{B}, v_{B}\right)_{C},
$$


and to investigate whether it is bounded below as a function of $B$. To do this we introduce a momentum cut-off in the fields and also approximate $v_{B}$ by a finite sum,

$$
v_{B}^{(n)}=\exp \frac{1}{2} \sum_{r=1}^{n}\left\langle q, w_{r}\right\rangle\left\langle q,(C-B) w_{r}\right\rangle,
$$

where $\left\{w_{r}\right\}_{r=1}^{n}$ are linearly independent test functions in $S$. If the space spanned by $\left\{w_{r}\right\}_{r=1}^{\infty}$ is dense in the completion of $S$ in both the $B$-norm and the $C$-norm, then formally

$$
v_{B}^{(n)} \rightarrow v_{B} \text { as } n \rightarrow \infty .
$$

This is the approximate $B$-vacuum, mentioned in the introduction. The form of $v_{B}^{(n)}$ is Gaussian and so the expectation value of the Hamiltonian $: H_{A L}:_{A}$ can easily be computed. We will find an upper bound for

$$
\inf _{n} \frac{\left(v_{B}^{(n)},: H_{A L}:_{A} v_{B}^{(n)}\right)_{C}}{\left(v_{B}^{(n)}, v_{B}^{(n)}\right)_{C}}
$$

which is independent of $C$ and recovers Coleman's bound.

\section{The Variational Argument}

The steps of the variational argument run as follows.

(1) We denote by $\phi_{\Lambda}(x)$ and $\pi_{\Lambda}(x)$ the quantum field operators with momentum cut-off $\Lambda$ at the space point $x$, constructed in the $C$-representation of the CCR on $L^{2}\left(Q, v_{C}\right)$. They are defined as follows. Choose a function $u$ in $S$ whose Fourier transform,

$$
\tilde{u}(k)=\int_{\mathbb{R}} e^{i k x} u(x) d x,
$$

approximates the characteristic function for the interval $[-\Lambda, \Lambda]$, and let $u_{x}$ denote the function

$$
u_{x}(y)=u(x-y) \text {. }
$$

Then, regarded as operators on $G \subseteq L^{2}\left(Q, v_{C}\right)$, it follows that

$$
\phi_{\Lambda}(x)=\phi_{u_{x}}
$$

and

$$
\pi_{\Lambda}(x)=-i \partial_{u_{x}}+i \phi_{C u_{x}}
$$

Now define

and

$$
\begin{aligned}
& \mathscr{H}_{\Lambda}^{0}(x)=\mathscr{H}^{0}\left(\phi_{\Lambda}(x), \pi_{\Lambda}(x)\right) \\
& \quad=\frac{1}{2}\left[-\partial_{u_{x}}^{2}+2\left(C u_{x}\right) \partial_{u_{x}}-\left(C u_{x}\right)^{2}+\left(\nabla u_{x}\right)^{2}+\left\langle u_{x}, C u_{x}\right\rangle\right]
\end{aligned}
$$

$$
: \mathscr{H}_{\Lambda}^{0}(x):_{A}=\mathscr{H}_{\Lambda}^{0}(x)-\mathscr{R}_{\Lambda}^{A}
$$


where

$$
\mathscr{R}_{A}^{A}=\frac{1}{4}\left\langle u_{x},\left[A-A^{-1} \nabla^{2}\right] u_{x}\right\rangle .
$$

Note that we have departed here from the usual procedure in constructive field theory, for we have defined the free Hamiltonian density as a function of the cut-off fields. Consequently, : $\mathscr{H}_{\Lambda}^{0}(x):_{A}$ is not local.

From the potential $\mathscr{V}$ we construct the cylinder function,

$$
\begin{aligned}
\mathscr{V}_{\Lambda}(x): Q & \rightarrow \mathbb{C} \\
q & \mapsto \lambda^{-2} \mathscr{V}\left(\lambda u_{x}(q)\right),
\end{aligned}
$$

whose Fourier transform we assume is of exponential type. Consequently, both $\mathscr{V}_{\Lambda}(x)$ and $: \mathscr{V}_{\Lambda}(x):_{A}$ lie in $G$ and define multiplication operators on $G$, which we denote by the same symbols. Note that we have included $\lambda$ in the definition of $\mathscr{V}_{\Lambda}$.

Under the hypotheses above, all of the operators $\mathscr{H}_{\Lambda}^{0}, \mathscr{V}_{A}, \mathscr{H}_{\Lambda},:_{H^{0}}^{0}:_{A}$, $: \mathscr{V}_{A}:_{A},: \mathscr{H}_{A}:_{A}$ are defined on $G$ and leave it invariant.

We now construct

$$
\begin{aligned}
& H_{\Lambda L}^{0}=\int_{-L}^{L} \mathscr{H}_{\Lambda}^{0}(x) d x, \\
& V_{\Lambda L}=\int_{-L}^{L} \mathscr{V}_{\Lambda}(x) d x,
\end{aligned}
$$

and the corresponding normally ordered forms, also with domain $G$.

(2) For the trial vector in the variational argument we choose

$$
v_{B}^{(n)}=\exp \frac{1}{2} \sum_{r, s=1}^{n}\left\langle q, w_{r}\right\rangle M_{r s}^{11}\left\langle q, w_{s}\right\rangle, \quad M_{r s}^{11}=M_{s r}^{11},
$$

in which we assume, without loss of generality, that the test functions $w_{1}, w_{2}, \ldots$, $w_{n}$ are linearly independent. We want to compute the expectation value of $: \mathscr{H}_{A}(x):_{A}$ for the state $v_{B}^{(n)}$, and this we can do provided that we can calculate

$$
\begin{aligned}
& \mathscr{X}^{(n)}=\left(v_{B}^{(n)}, v_{B}^{(n)}\right)_{C}, \\
& \mathscr{Y}^{(n)}=\left(v_{B}^{(n)},: \exp -i \lambda s u_{x}:_{A} v_{B}^{(n)}\right)_{C} /\left(v_{B}^{(n)}, v_{B}^{(n)}\right)_{C},
\end{aligned}
$$

and

$$
\mathscr{Z}^{(n)}=\left(v_{B}^{(n)},: \mathscr{H}_{A}^{0}(x):_{A} v_{B}^{(n)}\right)_{C} /\left(v_{B}^{(n)}, v_{B}^{(n)}\right)_{C}
$$

In order to calculate $\mathscr{X}^{(n)}$, we must integrate over the space spanned by $w_{1}$, $w_{2}, \ldots, w_{n}$. If we let

$$
N_{r s}^{11}=\left\langle w_{r}, w_{s}\right\rangle_{c}
$$

then Lemma 1 shows that

$$
\mathscr{X}^{(n)}=\operatorname{det}^{-1 / 2}\left(1-M^{11} N^{11}\right),
$$

and that the necessary and sufficient condition for $\mathscr{X}^{(n)}$ to be finite is

$$
\left[N^{11}\right]^{-1}-M^{11}>0 \text {. }
$$


Now

$$
\left(v_{B}^{(n)}, \exp \left[-i s \lambda u_{x}\right] v_{B}^{(n)}\right)_{C}=\int_{Q} \exp \left[\sum_{r, s}\left\langle q, w_{r}\right\rangle M_{r s}^{11}\left\langle q, w_{s}\right\rangle-i s \lambda\left\langle q, u_{x}\right\rangle\right] d v_{C}(q),
$$

so the integration is over the space spanned by $\left\{w_{1}, w_{2}, \ldots, w_{n}\right\}$ and $u_{x}$. In order to apply Lemma 1 , we set

$$
\begin{aligned}
& N=\left[\frac{\left\langle w_{r}, w_{s}\right\rangle_{C}}{\left\langle u_{x}, w_{s}\right\rangle_{C}} \mid \frac{\left\langle w_{r}, u_{x}\right\rangle_{C}}{\left\langle u_{x}, u_{x}\right\rangle_{C}}\right], \quad M=\left[\begin{array}{cc}
M^{11} & 0 \\
0 & 0
\end{array}\right] \\
& =\left(N^{i j}\right) \quad=\left(M^{i j}\right), \quad 1 \leqq i, j \leqq 2 \text {, }
\end{aligned}
$$

and

$$
z=\left[\begin{array}{c}
0 \\
-i s \lambda / 2
\end{array}\right]
$$

From Lemma 2 we have that the integral is convergent, given that (6.1) is satisfied, so

$$
\left(v_{B}^{(n)}, \exp \left[-i s \lambda u_{x}\right] v_{B}^{(n)}\right)_{C}=\exp (0,-i s \lambda / 2)\left[\begin{array}{l}
K^{11} K^{12} \\
K^{21} K^{22}
\end{array}\right]\left[\begin{array}{c}
0 \\
-i s \lambda / 2
\end{array}\right],
$$

where

$$
\begin{aligned}
K & =N(1-M N)^{-1} \\
& =\left[\begin{array}{lll}
N^{11}\left(1-M^{11} N^{11}\right)^{-1} & N^{11}\left(1-M^{11} N^{11}\right)^{-1} & M^{11} N^{12}+N^{12} \\
N^{21}\left(1-M^{11} N^{11}\right)^{-1} & N^{21}\left(1-M^{11} N^{11}\right)^{-1} & M^{11} N^{12}+N^{22}
\end{array}\right]
\end{aligned}
$$

Hence,

$$
\begin{aligned}
\mathscr{Y}^{(n)}= & \exp -\frac{s^{2} \lambda^{2}}{4}\left[\sum_{r, s}\left\langle u_{x}, w_{r}\right\rangle_{C}\left[\left(1-M^{11} N^{11}\right)^{-1} M^{11}\right]_{r s}\left\langle w_{s}, u_{x}\right\rangle_{C}\right. \\
& \left.+\left\langle u_{x}, u_{x}\right\rangle_{C}-\left\langle u_{x}, u_{x}\right\rangle_{A}\right] .
\end{aligned}
$$

Lastly, we must calculate $\mathscr{Z}^{(n)}$.

$$
\begin{aligned}
: \mathscr{H}_{\Lambda}^{0}(x):_{A} v_{B}^{(n)}(q)= & \frac{1}{2}\left[-\sum_{r, s, i, j}\left\langle u_{x}, w_{r}\right\rangle M_{r s}^{11}\left\langle u_{x}, w_{i}\right\rangle M_{i j}^{11}\left\langle q, w_{s}\right\rangle\left\langle q, w_{j}\right\rangle\right. \\
& -\sum_{r, s}\left\langle u_{x}, w_{r}\right\rangle M_{r s}^{11}\left\langle u_{x}, w_{s}\right\rangle \\
& +2 \sum_{r, s}\left\langle u_{x}, w_{r}\right\rangle M_{r s}^{11}\left\langle q, w_{s}\right\rangle\left\langle q, C u_{x}\right\rangle \\
& \left.-\left\langle q, C u_{x}\right\rangle^{2}+\left\langle q, \nabla u_{x}\right\rangle^{2}\right] \\
& \left.+\frac{1}{2}\left\langle u_{x}, C u_{x}\right\rangle-\frac{1}{4}\left\langle u_{x}, A u_{x}\right\rangle-\frac{1}{4}\left\langle\nabla u_{x}, A^{-1} \nabla u_{x}\right\rangle\right\} v_{B}^{(n)}(q), \\
= & \text { polynomial in } q \cdot v_{B}^{(n)}(q) .
\end{aligned}
$$


Thus, the calculation involves an integration over the space spanned by

$$
\left\{w_{1}, w_{2}, \ldots, w_{n}\right\}, u_{x}, \nabla u_{x} \text { and } C u_{x},
$$

so in the notation of Lemma 1 , we now set

$$
\begin{aligned}
N & =\left[\begin{array}{l|l|l|l}
\left\langle w_{r}, w_{s}\right\rangle_{C} & \left\langle w_{r}, u_{x}\right\rangle_{C} & \left\langle w_{r}, \nabla u_{x}\right\rangle_{C} & \left\langle w_{r}, C u_{x}\right\rangle_{C} \\
\hline\left\langle u_{x}, w_{s}\right\rangle_{C} & \left\langle u_{x}, u_{x}\right\rangle_{C} & \left\langle u_{x}, \nabla u_{x}\right\rangle_{C} & \left\langle u_{x}, C u_{x}\right\rangle_{C} \\
\hline\left\langle\nabla u_{x}, w_{s}\right\rangle_{C} & \left\langle\nabla u_{x}, u_{x}\right\rangle_{C} & \left\langle\nabla u_{x}, \nabla u_{x}\right\rangle_{C} & \left\langle\nabla u_{x}, C u_{x}\right\rangle_{C} \\
\hline\left\langle C u_{x}, w_{s}\right\rangle_{C} & \left\langle C u_{x}, u_{x}\right\rangle_{C} & \left\langle C u_{x}, \nabla u_{x}\right\rangle_{C} & \left\langle C u_{x}, C u_{x}\right\rangle_{C}
\end{array}\right] \\
& =\left(N^{i j}\right) \\
M & =\left[\begin{array}{ll|l|l|l|}
M^{11} & 0 & 0 & 0 \\
\hline 0 & 0 & 0 & 0 \\
\hline 0 & 0 & 0 & 0 \\
\hline 0 & 0 & 0
\end{array}\right] \\
& =\left(M^{i j}\right), 1 \leqq i, j \leqq 4 .
\end{aligned}
$$

Again, Lemma 2 assures us that the integral is finite, given that (6.1) is satisfied, and Lemma 1 shows that

$$
\begin{aligned}
Z^{(n)}= & -\frac{1}{4} \sum_{r, s}\left\langle u_{x}, w_{r}\right\rangle M_{r s}^{11} K_{s i}^{11} M_{i j}^{11}\left\langle w_{j}, u_{x}\right\rangle \\
& -\frac{1}{2} \sum_{r, s}^{i, j}\left\langle u_{x}, w_{r}\right\rangle M_{r s}^{11}\left\langle w_{s}, u_{x}\right\rangle \\
& +\frac{1}{2} \sum_{r, s}\left\langle u_{x}, w_{r}\right\rangle M_{r s}^{11} K_{r_{1}}^{14} \\
& +\frac{1}{4}\left\{K_{11}^{33}-K_{11}^{44}\right\}+\frac{1}{2}\left\langle u_{x}, C u_{x}\right\rangle-\frac{1}{4}\left\langle u_{x}, A u_{x}\right\rangle-\frac{1}{4}\left\langle\nabla u_{x}, A^{-1} \nabla u_{x}\right\rangle
\end{aligned}
$$

where

$$
K=N(1-M N)^{-1}
$$

After some manipulation and combination of terms, we obtain

$$
\begin{aligned}
\mathscr{Z}^{(n)}= & -\frac{1}{4} \sum_{r, s}\left\langle u_{x}, w_{r}\right\rangle M_{r s}^{11}\left\langle w_{s}, u_{x}\right\rangle \\
& +\frac{1}{4} \sum_{r, s}\left\langle\nabla u_{x}, w_{r}\right\rangle_{C}\left[\left(1-M^{11} N^{11}\right)^{-1} M^{11}\right]_{r s}\left\langle w_{s}, \nabla u_{x}\right\rangle_{C} \\
& +\frac{1}{4}\left(\left\|C u_{x}\right\|_{C}{ }^{2}+\left\|\nabla u_{x}\right\|_{C}{ }^{2}-\left\|A u_{x}\right\|_{A}{ }^{2}-\left\|\nabla u_{x}\right\|_{A}{ }^{2}\right) .
\end{aligned}
$$


Since $\mathscr{X}^{(n)}, \mathscr{Y}^{(n)}$ and $\mathscr{Z}^{(n)}$ only involve the leading blocks of $M$ and $N$, we can now dispense with the superscripts on $M$ and $N$.

We now make a particular choice for $M$, namely,

$$
M=N^{-1}-L^{-1}
$$

where

$$
L_{r s}=\left\langle w_{r}, w_{s}\right\rangle_{B} .
$$

Then condition (6.1) reduces to

$$
L^{-1}>0
$$

or, equivalently, that $B$ be a positive operator, which is certainly true. For this choice of $M$,

$$
(1-M N)^{-1} M=N^{-1} L N^{-1}-N^{-1} .
$$

If we now let $P^{B}$ and $P^{C}$ denote the $B$-orthogonal and $C$-orthogonal projections onto the span of $\left\{w_{1}, w_{2}, \ldots, w_{n}\right\}$, then we obtain

$$
\begin{aligned}
\mathscr{Y}^{(n)}= & \exp \frac{s^{2} \lambda^{2}}{4}\left[\left[\left\|u_{x}\right\|_{A}{ }^{2}-\left\|u_{x}\right\|_{B}{ }^{2}\right]+\left[\left\|u_{x}\right\|_{B}{ }^{2}-\left\|P^{C} u_{x}\right\|_{B}{ }^{2}-\left\|\left(1-P^{C}\right) u_{x}\right\|_{C}{ }^{2}\right]\right], \\
\mathscr{Z}^{(n)}= & \frac{1}{4}\left[\left\|B u_{x}\right\|_{B}{ }^{2}+\left\|\nabla u_{x}\right\|_{B}{ }^{2}-\left\|A u_{x}\right\|_{A}{ }^{2}-\left\|\nabla u_{x}\right\|_{A}{ }^{2}\right] \\
& +\frac{1}{4}\left[\left\|\left(1-P^{C}\right) C u_{x}\right\|_{C}{ }^{2}+\left\|\left(1-P^{C}\right) \nabla u_{x}\right\|_{C}{ }^{2}-\left\|\left(1-P^{B}\right) B u_{x}\right\|_{B}{ }^{2}-\left\|\nabla u_{x}\right\|_{B}{ }^{2}\right. \\
& \left.+\left\|P^{C} \nabla u_{x}\right\|_{B}{ }^{2}\right] .
\end{aligned}
$$

We assume that the subspace spanned by $\left\{w_{r}\right\}_{r=1}^{\infty}$ is dense in the completion of $S$ in the topologies of both the $B$-norm and the $C$-norm. Then both $P^{B}$ and $P^{C}$ will converge strongly to the identity in either topology. Thus,

$$
\begin{aligned}
\mathscr{Y}^{(\infty)} & =\lim _{n \rightarrow \infty} \mathscr{Y}^{(n)} \\
& =\exp \frac{s^{2} \lambda^{2}}{4}\left[\left\|u_{x}\right\|_{A}{ }^{2}-\left\|u_{x}\right\|_{B}{ }^{2}\right]
\end{aligned}
$$

and

$$
\begin{aligned}
\mathscr{Z}^{(\infty)} & =\lim _{n \rightarrow \infty} \mathscr{Z}^{(n)} \\
& =\frac{1}{4}\left[\left\|B u_{x}\right\|_{B}{ }^{2}-\left\|A u_{x}\right\|_{A}{ }^{2}+\left\|\nabla u_{x}\right\|_{B}{ }^{2}-\left\|\nabla u_{x}\right\|_{A}{ }^{2}\right] .
\end{aligned}
$$

Note that $\mathscr{Y}^{(\infty)}$ and $\mathscr{Z}^{(\infty)}$ are independent of $C$. We will see presently that $\mathscr{Y}^{(\infty)}$ and $\mathscr{Z}^{(\infty)}$ can be used to recover Coleman's bound.

(3) Now $\phi_{A}, \pi_{A}$ and $\mathscr{V}_{A}$ can be extended to the domain spanned by $v_{B}^{(n)}$ and $G$, because $\phi_{A}, \pi_{A}$ and $\mathscr{V}_{A}$ act upon $v_{B}^{(n)}$ by multiplication by a function in $G$, and functions in $G$ grow sufficiently slowly. Furthermore, these extensions remain symmetric, so $: \mathscr{H}_{A}:_{A}$ and $: H_{A L}:_{A}$ are also symmetric on the extended domain and

$$
\left(f,: H_{A L}:_{A} v_{B}^{(n)}\right)_{C}=\left(: H_{A L}:_{A} f, v_{B}^{(n)}\right)_{C}
$$


for all $f \in G$. The left hand side is a continuous function of $f$, because $: H_{A L}:_{A} v_{B}^{(n)}$ lies in $L^{2}\left(Q, v_{C}\right)$, so we conclude that $v_{B}^{(n)}$ lies in the domain of : $H_{A L}:{ }_{A}^{*}$.

We now assume that : $H_{A L}:_{A}$ is essentially self-adjoint on $G$. Then $v_{B}^{(n)}$ will lie in the domain of $: H_{A L}:_{A}$ and so we compute

$$
\begin{aligned}
F_{\lambda}^{(n)} & =\left(v_{B}^{(n)},: H_{A L}:_{A} v_{B}^{(n)}\right)_{C} /\left(v_{B}^{(n)}, v_{B}^{(n)}\right)_{C} \\
& =\int_{-L}^{L} d x\left[\mathscr{Z}^{(n)}+\frac{\lambda^{-2}}{2 \pi i} \int_{\Gamma} \mathscr{Y}^{(n)} \hat{\mathscr{V}}(s) d s\right]
\end{aligned}
$$

and

$$
\begin{aligned}
F_{\lambda}^{(\infty)} & =\int_{-L}^{L} d x\left[\mathscr{Z}^{(\infty)}+\frac{\lambda^{-2}}{2 \pi i} \int_{\Gamma} \mathscr{Y}^{(\infty)} \hat{\mathscr{V}}(s) d s\right] \\
& =2 L\left[\mathscr{Z}^{(\infty)}+\frac{\lambda^{-2}}{2 \pi i} \int_{\Gamma} \mathscr{Y}^{(\infty)} \hat{\mathscr{V}}(s) d s\right] .
\end{aligned}
$$

Finally, we observe that

$$
\inf _{n} \frac{\left(v_{B}^{(n)},: H_{\Lambda L}:{ }_{A} v_{B}^{(n)}\right)_{C}}{\left(v_{B}^{(n)}, v_{B}^{(n)}\right)_{C}} \leqq F_{\lambda}^{(\infty)}
$$

$F_{\lambda}^{(\infty)}$ is independent of $C$.

(4) The parameter $\beta$ appearing in $B$ is free, so if $F_{\lambda}^{(\infty)}$ can be made less than any prescribed bound as $\beta$ is varied, then the operator $: H_{A L}:_{A}$ must necessarily be unbounded below. As emphasised in the introduction, this still leaves the problem of verifying, in any particular model, that every cut-off Hamiltonian formed from $\mathscr{V}$ with this value of $\lambda$ must also be unbounded below. This would imply that any constructive approach to the model must fail, because a ground state could not exist.

\section{Examples and Applications}

In applying the preceding theory, we recall that

$$
\begin{aligned}
& A^{2}=-\nabla^{2}+\alpha^{2}, \\
& B^{2}=-\nabla^{2}+\beta^{2},
\end{aligned}
$$

in which $\alpha$ is fixed but $\beta$ is a free parameter which we may vary. Then, choosing $\tilde{u}$, the momentum cut-off, to be the characteristic function of the interval $[-\Lambda, \Lambda]$, we have

$$
\begin{aligned}
\mathscr{Y}^{(\infty)} & =\exp \frac{s^{2} \lambda^{2}}{4}\left[\frac{1}{2 \pi} \int_{-\Lambda}^{\Lambda} d k\left[\frac{1}{\sqrt{k^{2}+\alpha^{2}}}-\frac{1}{\sqrt{k^{2}+\beta^{2}}}\right]\right] \\
& =\left[\left(\frac{\sqrt{\Lambda^{2}+\alpha^{2}}+\Lambda}{\sqrt{\Lambda^{2}+\alpha^{2}}-\Lambda}\right)\left(\frac{\sqrt{\Lambda^{2}+\beta^{2}}-\Lambda}{\sqrt{\Lambda^{2}+\beta^{2}}+\Lambda}\right)\right]^{s^{2} \lambda^{2} / 8 \pi}
\end{aligned}
$$

and

$$
\mathscr{Z}^{(\infty)}=\frac{1}{8 \pi} \int_{-\Lambda}^{4} d k\left[\frac{2 k^{2}+\beta^{2}}{\sqrt{k^{2}+\beta^{2}}}-\frac{2 k^{2}+\alpha^{2}}{\sqrt{k^{2}+\alpha^{2}}}\right]
$$




$$
=\frac{\Lambda}{4 \pi}\left[\sqrt{\Lambda^{2}+\beta^{2}}-\sqrt{\Lambda^{2}+\alpha^{2}}\right] .
$$

Since we are only interested in large $A$, we will retain only the leading terms in the asymptotic developments of these functions, so

$$
\begin{aligned}
& \mathscr{Y}^{(\infty)}=\left(\beta^{2} / \alpha^{2}\right)^{\lambda^{2} s^{2} / 8 \pi}+0\left(\Lambda^{-1}\right), \\
& \mathscr{Z}^{(\infty)}=\frac{\beta^{2}-\alpha^{2}}{8 \pi}+0\left(\Lambda^{-1}\right) .
\end{aligned}
$$

Thus, we finally obtain

$$
F_{\lambda}^{(\infty)}=2 L\left[\frac{\beta^{2}-\alpha^{2}}{8 \pi}+\frac{\lambda^{-2}}{2 \pi i} \int\left(\frac{\beta^{2}}{\alpha^{2}}\right)^{s^{2} \lambda^{2} / 8 \pi} \hat{\mathscr{V}}(s) d s+0\left(\Lambda^{-1}\right)\right],
$$

which is the extension of Coleman's bound to general $\mathscr{V}$.

Example 1. Polynomial Potentials. We begin with this simple example in order to show that the two definitions we have introduced for the normally ordered form of a polynomial are consistent.

The real polynomial with even degree,

$$
\mathscr{V}(q)=\sum_{k=0}^{\ell} a_{k}\langle q, u\rangle^{k}, \quad a_{k} \in \mathbb{R},
$$

has the integral representation

$$
\mathscr{V}(q)=\frac{1}{2 \pi i} \int_{\Gamma} \hat{\mathscr{V}}(s) e^{-i s\langle q, u\rangle} d s,
$$

in which $\Gamma$ denotes a simple, anti-clockwise loop about the origin and

$$
\hat{\mathscr{V}}(s)=\sum_{k=0}^{\ell} a_{k} i^{k} k ! s^{-(k+1)} .
$$

According to the definition,

$$
\begin{aligned}
: \mathscr{V}_{A}(x):(q) & =\frac{\lambda^{-2}}{2 \pi i} \int \hat{\Gamma}(s): e^{-i \lambda s u_{x}}:_{A}(q) d s \\
& =\frac{\lambda^{-2}}{2 \pi i} \int_{\Gamma}^{\ell} \sum_{k=0}^{\ell} i^{k} \frac{a_{k} k !}{s^{k+1}} e^{-i \lambda s\left\langle q, u_{x}\right\rangle+\left(\lambda^{2} s^{2} / 4\right)\left\langle u_{x}, u_{x}\right\rangle_{A}}, \\
& =\lambda^{-2} \sum_{k=0}^{\ell} a_{k} i^{k}\left[\left(\frac{d}{d s}\right)^{k} e^{-i \lambda s\left\langle q, u_{x}\right\rangle+\left(\lambda^{2} s^{2} / 4\right)\left\langle u_{x}, u_{x}\right\rangle_{A}}\right]_{s=0} \\
& =\lambda^{-2} \sum_{k=0}^{\ell} a_{k} \lambda^{k} \sum_{m=0}^{[k / 2]} \frac{k !(-1)^{m}}{m !(k-2 m) !}\left\langle q, u_{x}\right\rangle^{k-2 m}\left\|u_{x}\right\|_{A}^{2 m} 4^{-m},
\end{aligned}
$$

which agrees with the conventional definition. 
We find that

$$
F_{\lambda}^{(\infty)}=2 L\left[\frac{\beta^{2}-\alpha^{2}}{8 \pi}+\lambda^{-2} \sum_{j=0}^{[\ell / 2]} a_{2 j}(-\rho)^{j} \frac{(2 j) !}{j !}+0\left(\Lambda^{-1}\right)\right],
$$

where $\rho=\frac{\lambda^{2}}{8 \pi} \log \left(\beta^{2} / \alpha^{2}\right) . F_{\lambda}^{(\infty)}$ is bounded below for all $\beta$.

Example 2. Trigonometric Potentials. Suppose that $\mathscr{V}$ has the form

$$
\mathscr{V}(q)=\sum_{k=1}^{\ell}\left(a_{k} e^{-i s_{k}\langle q, u\rangle}+\bar{a}_{k} e^{+i \bar{s}_{k}\langle q, u\rangle}\right) .
$$

If the numbers $s_{k}$ are real, then $f$ is a trigonometric polynomial, but we will admit complex values for $s_{k}$ so that this class also includes exponential functions and combinations of exponential and trigonometric polynomials. If

$$
s_{k}=\sigma_{k}+i \tau_{k},
$$

and

$$
a_{k}=\left|a_{k}\right| e^{i \theta_{k}}
$$

then

$$
\mathscr{V}(q)=\sum_{k=0}^{\ell} 2\left|a_{k}\right| e^{\tau_{k}\langle q, u\rangle} \cos \left(\sigma_{k}\langle q, u\rangle-\theta_{k}\right) .
$$

Such functions can be represented in the form

$$
\mathscr{V}(q)=\frac{1}{2 \pi i} \int_{\Gamma} \hat{\mathscr{V}}(s) e^{-i s\langle q, u\rangle} d s,
$$

provided we let $\Gamma$ be a simple anti-clockwise contour which encircles $s_{k},-\bar{s}_{k}$, $k=0, \ldots, \ell$, and choose

$$
\hat{\mathscr{V}}(s)=\sum_{k=0}^{\ell}\left(\frac{a_{k}}{s-s_{k}}+\frac{\bar{a}_{k}}{s+\bar{s}_{k}}\right) .
$$

This general form includes potentials such as $e^{\lambda \phi}$ for which we find that $\lambda$ is unrestricted; $\cos ^{2} \lambda \phi$ for which we must have $\lambda^{2} \leqq 2 \pi$; and $e^{c \lambda \phi} \cos ^{2} \lambda \phi$ for which there is no restriction on $\lambda$ provided $c^{2} \geqq 4$, but otherwise we need $\left(4-c^{2}\right) \lambda^{2} \leqq 8 \pi$. In general we obtain

$$
F_{\lambda}^{(\infty)}=2 L\left[\frac{\beta^{2}-\alpha^{2}}{8 \pi}+\lambda^{-2} \sum_{k=0}^{\ell} 2\left|a_{k}\right| e^{\rho\left(\sigma_{k}^{2}-\tau_{k}^{2}\right)} \cos \left(2 \sigma_{k} \tau_{k} \rho+\theta_{k}\right)\right],
$$

where

$$
\rho=\frac{\lambda^{2}}{8 \pi} \log \beta^{2} / \alpha^{2}
$$

If $\sigma_{k} \tau_{k} \neq 0$, we can choose values of $\beta^{2}$, small or large, such that $\cos \left(2 \sigma_{k} \tau_{k} \rho+\theta_{k}\right)$ is negative. The behaviour of $F_{\lambda}^{(\infty)}$ depends on the relative values of $s_{k}$ and $a_{k}$. 
For example, if $\mathscr{V}$ consists of a single term with:

(1) $\sigma_{k}^{2}=\tau_{k}^{2}$, then $F_{\lambda}^{(\infty)}$ is bounded below;

(2) $\sigma_{k}^{2}>\tau_{k}^{2}$, then $F_{\lambda}^{(\infty)}$ is unbounded below unless $\lambda^{2}\left(\sigma_{k}^{2}-\tau_{k}^{2}\right) \leqq 8 \pi$;

(3) $\sigma_{k}^{2}<\tau_{k}^{2}$, then $F_{\lambda}^{(\infty)}$ is unbounded below for all non-zero $\lambda$.

In general, terms in $F_{\lambda}^{(\infty)}$ can be either positive or negative for large and small $\beta^{2}$, and the combined result depends on the explicit form for $\mathscr{V}$.

Example 3. Combined Trigonometric and Polynomial Functions. It should be clear that we can obtain any combination of polynomial, exponential and trigonometric functions by choosing $\hat{\mathscr{V}}$ to be meromorphic with a finite number of poles and $\Gamma$ a contour which encloses these poles. When we form potentials by adding polynomials and trigonometric functions, we find that the behaviour of $F_{\lambda}^{(\infty)}$ is governed by the latter, since polynomials, according to (7.2), contribute terms merely logarithmic in $\beta^{2}$. Much the same is true if we form potentials by multiplying trigonometric and polynomial functions. For example, a potential of the form $p(\lambda \phi) \cos ^{2} \lambda \phi$, where $p$ is an even, non-negative polynomial, must satisfy $\lambda^{2} \leqq 2 \pi$ if $p$ has degree $0,2,6,10, \ldots$, but otherwise there is no restriction on $\lambda$.

Example 4. Rational Functions. If $\mathscr{V}$ is a rational function whose square is integrable along the real line, then we find that the Fourier transform of $\mathscr{V}$ (considered as a function on configuration space) is an entire function whose order of growth exceeds one. Such a potential does not have an integral representation over a bounded contour $\Gamma$ with the exponential function as kernel. According to our program, such a potential cannot be normally ordered and must be rejected.

We can arrive at this result in another way. We can write

$$
\mathscr{V}(t)=\frac{1}{2 \pi} \int_{-\infty}^{\infty} e^{-i s t} \widehat{\mathscr{V}}(s) d s,
$$

where $\hat{\mathscr{V}}$ is the usual Fourier transform, and perhaps define

and

$$
\mathscr{V}(q)=\frac{1}{2 \pi} \int_{-\infty}^{\infty} e^{-i s\langle q, u\rangle} \hat{\mathscr{V}}(s) d s
$$

$$
\begin{aligned}
: \mathscr{V}_{\Lambda}(x):_{A}(q) & =\frac{\lambda^{-2}}{2} \int_{-\infty}^{\infty}: e^{-i s u_{x}}:_{A}(q) \hat{\mathscr{V}}(s) d s \\
& =\frac{\lambda^{-2}}{2 \pi} \int_{-\infty}^{\infty} e^{-i s \lambda\left\langle q, u_{x}\right\rangle} e^{1 / 4 \lambda^{2} s^{2}\left\|u_{x}\right\|^{2}} \hat{A}(s) d s .
\end{aligned}
$$

However, the Fourier transform $\hat{\mathscr{V}}$ of a rational function generally will decay like $e^{-a s}$, so the integral in (7.3) cannot converge. Again we must discard rational potentials because they cannot be normally ordered.

Acknowledgement. The authors would like to thank Prof. C. A. Hurst for penetrating criticism.

\section{References}

1. Coleman, S. : Phys. Rev. D11, 2088-2097 (1975)

2. Epstein, H., Glaser, V., Jaffe, A. : Non-positivity of the energy density in quantised field theories. Nuovo Cimento 36, 1016-1022 (1965) 
3. Glimm, J., Jaffe, A. : Boson quantum field models, in mathematics of contemporary physics, Streater, R. F. (ed.) New York: Academic Press 1972

4. Chodos, A., Klein, A. : Phys. Rev. D14, 1663-1666 (1976)

5. Gel'fand, I. M., Vilenkin, N. Ya. : Generalised Functions, Vol. 4, Chap. IV. New York: Academic Press 1964

6. Gel'fand, I. M., Shilov, G. E. : Generalised Functions, Vol. 2, Chap. IV. New York: Academic Press 1968

7. Ronkin, L. I. : Introduction to the theory of entire functions of several variables. Translations of mathematical monographs, Vol. 74, Providence, Rhode Island: American Mathematical Society, 1974

8. Hфegh-Krohn, R. : Commun. Math. Phys. 21, 244-255 (1971)

9. Fröhlich, J. : Quantised "Sine-Gordon" equation with a non-vanishing mass term in two spacetime dimensions. Phys. Rev. Lett. 34, 833-836 (1975)

10. Skagerstam, B. S. : Lett. in Math. Phys. 1, 499-503 (1977)

11. Skagerstam, B. S. : Nucl. Phys. B131, 421-428 (1977)

Communicated by K. Osterwalder

Received October 15, 1980; in revised form March 31, 1981

Note added in proof. B. S. Skagerstam $[10,11]$ has applied Coleman's trick in order to study quantum corrections to classical confinement. 